\title{
Order-Disorder Transition of Symmetric Poly(styrene-b-2-vinylpyridine) in Bulk and Solution
}

\author{
Yoshiaki Takahashi, ${ }^{\dagger}$ Shinichi Kitade, Masahiro Noda, Nobuo OchiaI, \\ Ichiro NodA, Masayuki IMAI, ${ }^{*}$ and Yushu Matsushita* \\ Department of Applied Chemistry, Graduate School of Engineering, \\ Nagoya University, Nagoya 464-8603, Japan \\ * Neutron Scattering Laboratory, The Institute for Solid State Physics, \\ The University of Tokyo, Shirakata 106-1, Tokai, Naka 319-1106, Japan
}

(Received August 29, 1997)

\begin{abstract}
Order-disorder transitions (ODT) of symmetric poly(styrene- $b$-2-vinylpyridine) in a common good solvent and bulk were determined by dynamic rheological and small-angle neutron scattering measurements. ODT conditions determined by both methods are consistent with each other and the degree of polymerization dependence of critical volume fraction in solution at almost constant temperature agrees with the theoretical prediction for semidilute solutions. However, semidilute behavior persists to a much higher concentration than for ordinary polymer solutions and the crossover from semidilute to concentrated regimes is not clear.

KEY WORDS Order-Disorder Transition / Symmetric Diblock Copolymers / Poly(styrene- $b-2$ vinylpyridine) / Small-Angle Neutron Scattering / Semidilute Region /
\end{abstract}

Block copolymers exhibit a phase transition between ordered (microphase-separated) and disordered (homogeneous) states under certain conditions. The orderdisorder transitions (ODT) of block copolymers have been extensively studied and the results are summarized in the literature. ${ }^{1-8} \mathrm{~A}$ brief outline of the results is as follows.

Theoretically, the phase diagram for diblock copolymers in bulk was first discussed by the mean-field approach, ${ }^{9}$ and later modified to include fluctuation effects. ${ }^{10}$ According to the theory, the ODT of symmetric diblock copolymers occurs at

$$
(\chi N)_{\mathrm{ODT}}=10.5+41.0 \bar{N}^{-1 / 3}
$$

where $\chi$ is the Flory-Huggins interaction parameter, $N$ is the number of statistical segments (monomers) per copolymer molecule and

$$
\bar{N}=\left(6\left\langle S^{2}\right\rangle / M_{n}\right)^{3} M_{n} \rho^{2} N_{\AA}^{2}
$$

is a term for fluctuation correction. Here $\left\langle S^{2}\right\rangle, M_{n}, \rho$, and $N_{\mathrm{A}}$ are the mean square radius of gyration, number-average molecular weight, mass density and Avogadro's number, respectively. It was also predicted that eq 1 and 2 can be applied to block copolymer solutions by appropriate replacement of the parameters. ${ }^{11,12}$

Experimentally, most studies on ODT have been carried out for polystyrene-polydiene diblock copolymers such as poly(styrene- $b$-isoprene) (SI) and hydrocarbon diblock copolymers such as poly(ethylenepropylene- $b$-ethylethylene) in bulk. Several experimental methods to determine ODT have been examined. At present, small-angle X-ray scattering, small-angle neutron scattering (SANS), birefringence measurement and dynamic rheological measurements (DRM) are generally used. ${ }^{1-8}$

Recently, we studied the viscoelastic properties of SI diblock copolymer solutions near ODT and reported that the shear rate dependence of the first normal stress difference changes from first order to second order, after shear-induced alignment of the microdomain structure occurs. ${ }^{13}$ Generally speaking, systematic studies on viscoelastic properties, especially those under steady shear flow are much easier in solution than in bulk. Difference in viscoelastic properties of block components may affect the shear-induced alignment. For better understanding of the viscoelastic properties and shearinduced alignment of diblock copolymers near ODT, it is essential to study them both in bulk and solutions using well-characterized diblock copolymers which consist of block components having similar viscoelastic properties in comparison with those having different viscoelastic properties such as SI.

Polystyrene (PS) and poly(2-vinylpyridine) (P2VP) are suitable block components for such study because they have very similar Kuhn segment length ${ }^{14}$ and similar viscoelastic properties in bulk and a common good solvent. ${ }^{15}$ Matsushita et al., ${ }^{16-22}$ studied the preparation, characterization, morphology and chain conformation for poly(styrene- $b$-2-vinylpyridine) (SP) and the deuterated analogue, i.e., poly(styrene- $d_{8}-b$-2-vinylpyridine) (DP) with different compositions, molecular weights, and narrow molecular weight distributions.

To study viscoelastic properties near ODT, it is necessary to know ODT conditions. Very recently, Bates et al., ${ }^{8}$ reported ODT of SP and DP diblock copolymers in bulk by DRM and SANS. ODT has been studied mainly in bulk not only for SP and DP diblock copolymers but also for other block copolymers as already mentioned. A few studies ${ }^{3,23,24}$ were carried out in "neutral solvents" at relatively high concentrations but not in common good solvents. In this work, we studied ODT for symmetric SP and DP diblock copolymers with narrow molecular weight distributions in a common good solvent over a wide range of concentration as well as in bulk by SANS and DRM.

\footnotetext{
$\uparrow$ To whom correspondence should be addressed.
} 
Table I. Molecular characteristics of poly(styrene- $b$-2-vinylpyridine)s

\begin{tabular}{lrrrc}
\hline $\begin{array}{c}\text { Sample } \\
\text { code }\end{array}$ & $10^{-4} \mathrm{M}$ & $\begin{array}{c}M_{w} / M_{n} \\
(\mathrm{GPC})\end{array}$ & $\phi_{\mathrm{Ps}}{ }^{\mathrm{e}}$ & $\begin{array}{c}T_{\mathrm{g}} /{ }^{\circ} \mathrm{C} \\
(\mathrm{DSC})\end{array}$ \\
\hline $\mathrm{SP} 23$ & $109^{\mathrm{a}}$ & 1.03 & 0.53 & \\
$\mathrm{SP} 12$ & $38.8^{\mathrm{b}}$ & 1.03 & 0.52 & 101 \\
$\mathrm{DP} 20$ & $36.0^{\mathrm{a}}$ & 1.08 & 0.53 & \\
DP19 & $20.0^{\mathrm{c}}$ & 1.05 & 0.52 & 95 \\
$\mathrm{SP} 22$ & $3.8^{\mathrm{b}}$ & 1.01 & 0.52 & \\
$\mathrm{SP} 29$ & $1.5^{\mathrm{d}}$ & 1.12 & 0.50 & 91 \\
DP17 & $1.2^{\mathrm{d}}$ & 1.05 & 0.52 & 95 \\
\hline
\end{tabular}

${ }^{a}$ Weight-average molecular weights $M_{w}$ determined by LS. ${ }^{\mathrm{b}} \mathrm{Num}$ ber-average molecular weights $M_{n}$ determined by osmometry. ${ }^{\mathrm{c}} M_{n}$ determined by GPC. ${ }^{\mathrm{d}} M_{n}$ determined by GPC-MALLS. ${ }^{\mathrm{e}}$ Volume fraction of PS determined by elemental analysis or pyrolysis-gas chromatography.

\section{EXPERIMENTAL}

\section{Materials}

Molecular characteristics of the samples used in this work are listed in Table I. SP samples except SP29 were prepared and characterized in previous works. ${ }^{16-22}$ SP29 and DP samples were prepared in this work in the same manner as in the previous work. ${ }^{16,17}$ The molecular weight of DP20 was measured by light scattering in the same manner as in the previous work. ${ }^{17}$ The molecular weights of SP29 and DP17 were measured by gel permeation chromatography (GPC)-multi-angle laser light scattering method in tetrahydrofuran with a DAWN F of Wyatt Technology Corp. Refractive index increment $(\mathrm{d} n / \mathrm{d} c)$ value of the PS solution was used, since PS and P2VP have about the same $\mathrm{d} n / \mathrm{d} c .{ }^{17}$ The molecular weight of DP19 was determined by ordinary GPC using a calibration curve made from the data of other samples, since it was slightly colored. Glass transition temperatures, $T_{\mathrm{g}}$, of some samples were measured with a differential scanning calorimeter DSC 3100 of MAC Science Co., with a constant heating rate, $5^{\circ} \mathrm{C} \mathrm{min}^{-1}$. The solvent used was $\alpha$-chloronaphthalene $(\alpha \mathrm{CN})$, a common good solvent for both block components. ${ }^{15}$

\section{Rheological Measurements}

Dynamic rheological measurements were performed under oscillatory shear flow with a Mechanical Spectrometer RMS800 and an ARES with high sensitivity transducers of Rheometric Scientific Inc. The instruments and geometries (cone-plate and parallel-plate) were chosen appropriately to measure stress at low frequency to determine ODT conditions from the differences in the frequency $(\omega)$ dependence of the storage $\left(G^{\prime}\right)$ and loss $\left(G^{\prime \prime}\right)$ moduli. The highest strain used for bulk samples was $5 \%$ and that for solutions was $15 \%$. All the data were reproductive in repeated measurements.

\section{$S A N S$}

SANS measurements were performed with SANS-U spectrometer at the Neutron Scattering Laboratory of the Institute for Solid State Physics, The University of Tokyo in Tokai, Ibaraki, Japan. A film of DP17, annealed for one week at $95^{\circ} \mathrm{C}$, which is lower than the ODT determined for the as-cast film, ${ }^{25}$ but higher than the $T_{\mathrm{g}}$, and its highly concentrated $\alpha \mathrm{CN}$ solutions, each cast from tetrahydrofuran (THF) solution and moulded to a disk, were measured over a wide range of temperature. DP19 and DP20 in $\alpha \mathrm{CN}$ at different concentrations near ODT determined by DRM were measured in a limited range of temperature around $30^{\circ} \mathrm{C}$. The wavelength, $\lambda$, was $0.7 \mathrm{~nm}$ and the sample to detector distance was $3.0 \mathrm{~m}$ for DP17, while $12 \mathrm{~m}$ for DP19 and DP20. Measurements were always started from the lowest temperature.

The scattered intensity obtained on a two-dimensional detector was corrected for background and incoherent scattering of the component polymers and solvent, though incoherent scattering of polymers was neglected for the solutions at low concentrations. Intensity was circularly averaged and the data of DP17 were converted to absolute intensity using a secondary standard sample.

\section{RESULTS}

\section{Rheological Measurements}

Figure 1 shows double logarithmic plots of master curves of $G^{\prime}$ and $G^{\prime \prime}$ against $\omega a_{\mathrm{T}}$ for DP17 (1a) and SP29 (1b) in bulk, where $a_{\mathrm{T}}$ is the temperature shift factor. The reference temperature is $160^{\circ} \mathrm{C}$. It is clear that $G^{\prime}$ and $G^{\prime \prime}$ of DP17 (Figure 1a) are proportional to $\omega$ and $\omega^{2}$, respectively, in a wide range of $\omega$ at all the temperatures. This is typical behavior of homogeneous polymers in the terminal region. ODT temperatures, $T_{\mathrm{ODT}}$, of DP17 in bulk and solution could not be determined by DRM since they are close to $T_{\mathrm{g}}$ of the samples, so that they were only determined by SANS as described later.

As shown in Figure 1 b, most $G^{\prime}$ and $G^{\prime \prime}$ data for SP29 exhibit the same $\omega$ dependencies as DP17 in bulk, except for $G^{\prime}$ data at 131 and $135^{\circ} \mathrm{C}$, which deviate from the master curve at low $\omega$, implying that these temperatures are lower than $T_{\mathrm{ODT}}{ }^{1-5}$ Therefore, $T_{\mathrm{ODT}}$ of SP29 is in between 135 and $138^{\circ} \mathrm{C}$.

Figure 2 shows double logarithmic plots of $G^{\prime}$ and $G^{\prime \prime}$ against $\omega$ for DP19 in $\alpha \mathrm{CN}$ with different polymer volume fractions $\phi$ at constant temperature, $28^{\circ} \mathrm{C}$. $G^{\prime \prime}$ data for all solutions are proportional to $\omega$. $G^{\prime}$ data of the solutions with $\phi=0.151$ and 0.164 are proportional to $\omega^{2}$, while $\omega$ dependency of other solutions becomes lower at the low $\omega$ regions, indicating the behavior of ordered state. From differences in $G^{\prime}$ behavior, the critical value of $\phi$ for ODT, $\phi_{\mathrm{ODT}}$, of DP19 solution at $28^{\circ} \mathrm{C}$ was determined to be between 0.164 and 0.185 . For all the other solutions, $T_{\mathrm{ODT}}$ at constant $\phi$, and/or $\phi_{\mathrm{ODT}}$ at constant temperature were determined in the same manner and are tabulated in Table II, as a range of two adjacent data points in which $T_{\mathrm{ODT}}$ or $\phi_{\mathrm{ODT}}$ is located.

\section{SANS}

Figures 3 and 4 show some examples of plots of scattered intensity, $I(q)$, against magnitude of scattering vector, $q$, where $q=(4 \pi / \lambda) \sin (\theta / 2)$ and $\theta$ is scattering angle, for DP19 (Figure 3a) and DP20 (3b) in $\alpha \mathrm{CN}$, and for the annealed bulk sample of DP17, respectively. In Figure 3, scattered intensity curves significantly differ in magnitude by small change of temperature and/or $\phi$, so that we can easily determine $T_{\mathrm{ODT}}$ and $\phi_{\mathrm{ODT}}$ for these 

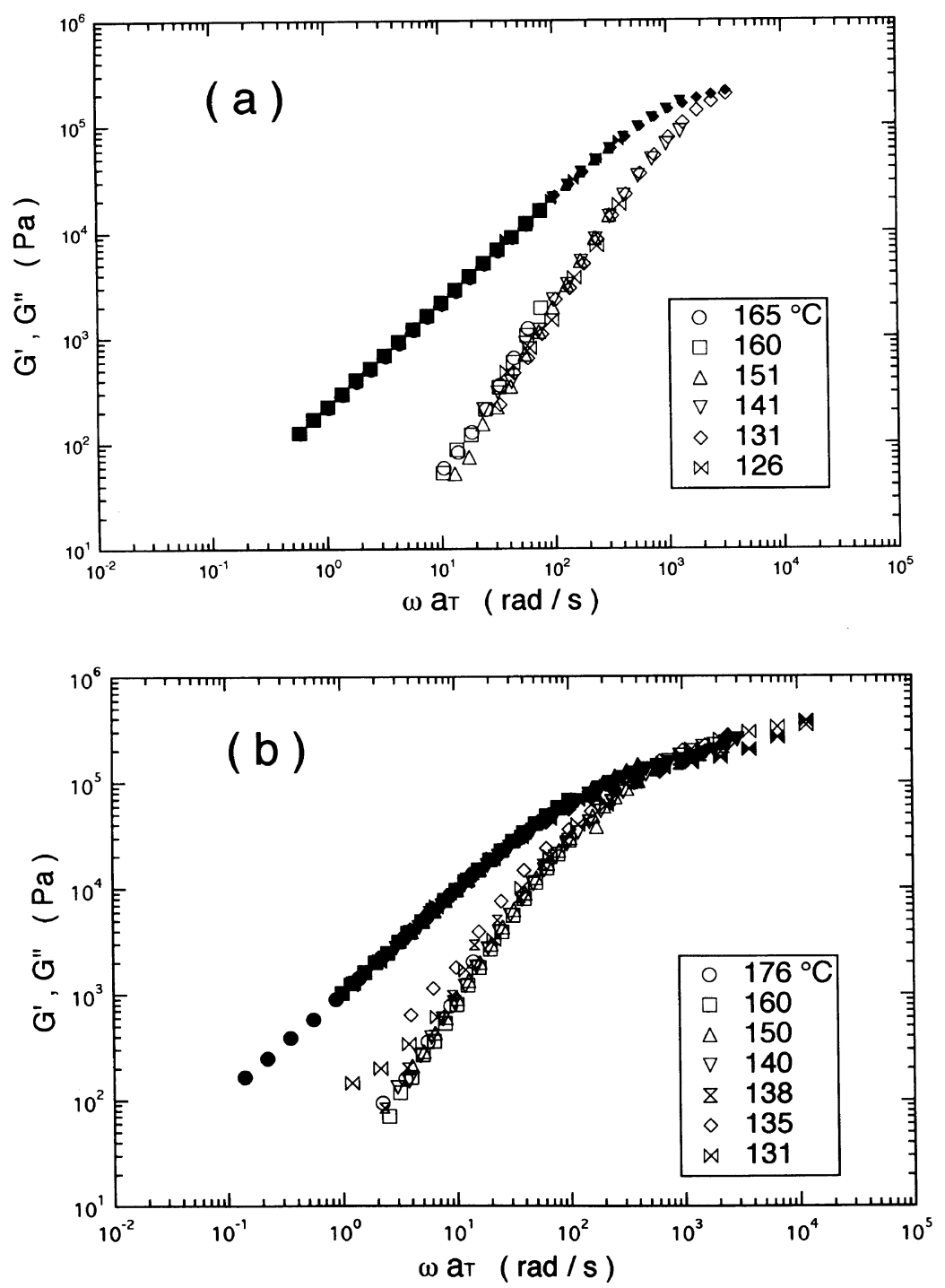

Figure 1. Double logarithmic plots of $G^{\prime}$ (open symbols) and $G^{\prime \prime}$ (filled symbols) against $\omega a_{\mathbf{T}}$ for (a) DP17 and (b) SP29 in bulk at $160^{\circ} \mathrm{C}$. Symbols are denoted in the figure.

solutions; $T_{\mathrm{ODT}}$ is in between 28.5 and $30^{\circ} \mathrm{C}$ at $\phi=0.180$ for DP19 and $\phi_{\mathrm{ODT}}$ is in between 0.111 and 0.122 at $30^{\circ} \mathrm{C}$ for DP20, consistent with values determined by DRM.

In Figure 4, the scattered intensity for DP17 in bulk explicitly changes between 93 and $102^{\circ} \mathrm{C}$ so that $T_{\mathrm{ODT}}$ is located in this temperature range. However, change in scattered intensity is not so significant as in Figure 3. The situation is the same or even less apparent for DP17 solution. Therefore, in addition to the change in scattered intensity, characterized by the peak value of the intensity, $I^{*}, q$ of the peak position, $q^{*}$, are analyzed to determine $T_{\mathrm{ODT}}$ in these cases.

To obtain $I^{*}$ and $q^{*}$, all data including those in the ordered state (filled symbols) are fitted to the theoretical function presented by Leibler ${ }^{9}$ as shown by solid lines in Figure 4. Various corrections ${ }^{26}$ such as desmearing are needed for quantitative discussions on fitting to the theory in the disordered state. The scattering profile in the ordered state cannot be discussed by the theory of Leibler. As can be seen in the figure, the fitted theoretical curves approximately represent overall shape of scattered profile at all the temperatures and $q^{*}$ become almost the same for smeared and desmeared data, ${ }^{5}$ so that these curves can be used as a guide to fairly determine $I^{*}$ and $q^{*}$.

Figure 5 shows plots of $I^{*-1}$ and $D\left(D \equiv 2 \pi / q^{*}\right)$ against $\left(T / T_{\text {ODT }}\right)^{-1}$ for DP17 in bulk and solution with $\phi=0.95$, 0.90 and 0.83 . As described below, $T_{\mathrm{ODT}}$ of the $\alpha \mathrm{CN}$ solutions of DP17 were synthetically determined in comparison with bulk behavior of $I^{*-1}$ and $D$.

As shown in Figure 5, the $I^{*-1}$ data of annealed bulk sample (filled circle) linearly decrease with increase of $T^{-1}$ at low $T^{-1}$ (i.e., high $T$ ), but $T^{-1}$ dependence of $I^{*-1}$ becomes weaker with increase of $T^{-1}$ and finally becomes almost constant after small discontinuity due to ODT, observed at around $10^{3} T^{-1}=2.7$ (indicated by a vertical solid line at $\left.\left(T / T_{\mathrm{ODT}}\right)^{-1}=1\right) . D$ of the annealed bulk sample are almost constant at low $T^{-1}$, but increase between $10^{3} T^{-1}=2.5$ and 2.7 (or $\left(T / T_{\mathrm{ODT}}\right)^{-1}=0.93$ and 1 ), as observed in Figure 5b. At the higher $T^{-1}$ (i.e., lower $T), D$ again become almost constant at approximately $11.2 \mathrm{~nm}$, consistent with $D$ reported for symmetric SP diblock copolymers in the strong segregation limit determined by desmeared data of small-angle X-ray scattering. ${ }^{19}$ The increase of $D$ before reaching $T_{\mathrm{ODT}}$ has been reported in other studies, ${ }^{5,7}$ and was 


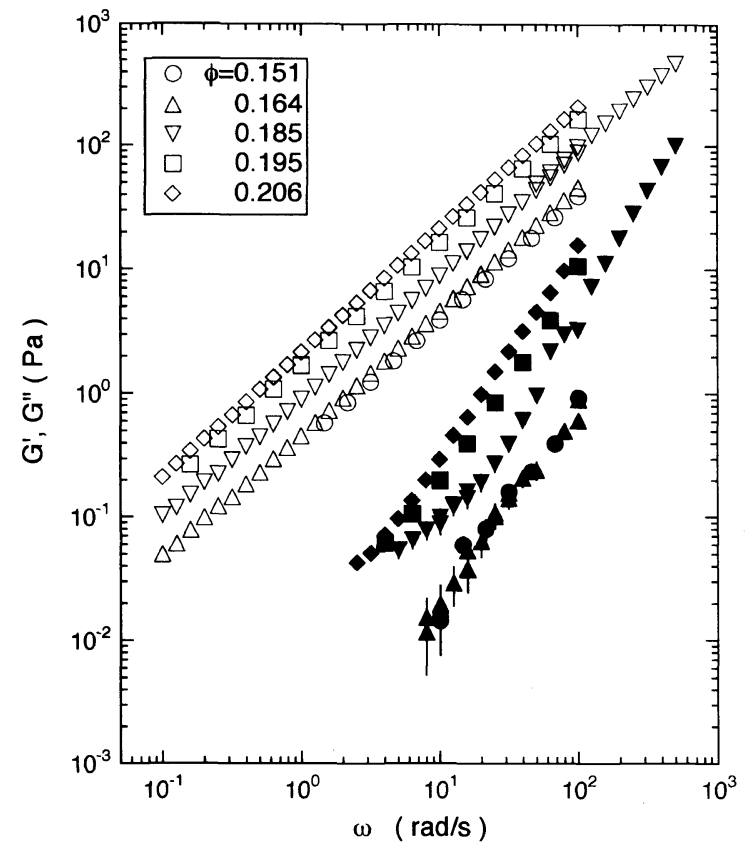

Figure 2. Double logarithmic plots of $G^{\prime}$ and $G^{\prime \prime}$ against $\omega$ for DP19 solutions at $28^{\circ} \mathrm{C}$. Volume fractions of polymer $\phi$ are denoted in the figure.

Table IIa. $\phi_{\text {ODT }}$ determined at constant $T$

\begin{tabular}{lcccc}
\hline $\begin{array}{c}\text { Sample } \\
\text { code }\end{array}$ & $N_{n}{ }^{*}$ & $\phi_{\text {ODT }}$ & $T /{ }^{\circ} \mathrm{C}$ & Method \\
\hline SP23 & $1.01 \times 10^{4}$ & $0.063-0.076$ & 30 & (a) \\
SP12 & $3.70 \times 10^{3}$ & $0.110-0.130$ & 30 & (a) \\
DP20 & $3.06 \times 10^{3}$ & $0.111-0.122$ & 30 & SANS \\
& & $0.122-0.133$ & 30 & (a) \\
DP19 & $1.75 \times 10^{3}$ & $0.164-0.185$ & 28 & (a) \\
SP22 & $3.62 \times 10^{2}$ & $0.453-0.467$ & 22 & (a) \\
\hline
\end{tabular}

* Number-average degree of polymerization. (a) By rheology.

Table IIb. $T_{\mathrm{ODT}}$ determined at constant $\phi$

\begin{tabular}{cccc}
\hline $\begin{array}{c}\text { Sample } \\
\text { code }\end{array}$ & $\phi$ & $T_{\mathrm{ODT}} /{ }^{\circ} \mathrm{C}$ & Method \\
\hline DP19 & 0.180 & $28.5-30$ & SANS \\
SP22 & 0.500 & $33-37$ & (a) \\
SP29 & 1 & $135-138$ & (a) \\
DP17 & 1 & $93-102$ & SANS \\
& 0.950 & $64-71$ & SANS \\
& 0.900 & $57-61$ & SANS \\
& 0.830 & $46-49$ & SANS \\
\hline
\end{tabular}

(a) By rheology.

explained by the fluctuation effect. The discontinuity of the $I^{*-1}$ data and change in $T^{-1}$ dependence of $D$ are consistent.

Similarly, $T_{\mathrm{ODT}}$ of DP17 solutions were determined from change in $T^{-1}$ dependencies of $I^{*-1}$ and $D$, though the discontinuity of $I^{*-1}$ is somewhat unclear in comparison with that of DP17 in bulk. $T_{\mathrm{ODT}}$ of DP17 solutions with $\phi=0.95,0.90$, and 0.83 thus determined are 64 to $71^{\circ} \mathrm{C}, 57$ to $61^{\circ} \mathrm{C}$, and 46 to $49^{\circ} \mathrm{C}$, respectively. All ODT conditions determined by SANS are tabulated in Table II.

Hereafter, we express the median value of the range in which $T_{\mathrm{ODT}}$ or $\phi_{\mathrm{ODT}}$ is located (Table II) as $T_{\mathrm{ODT}}$ or
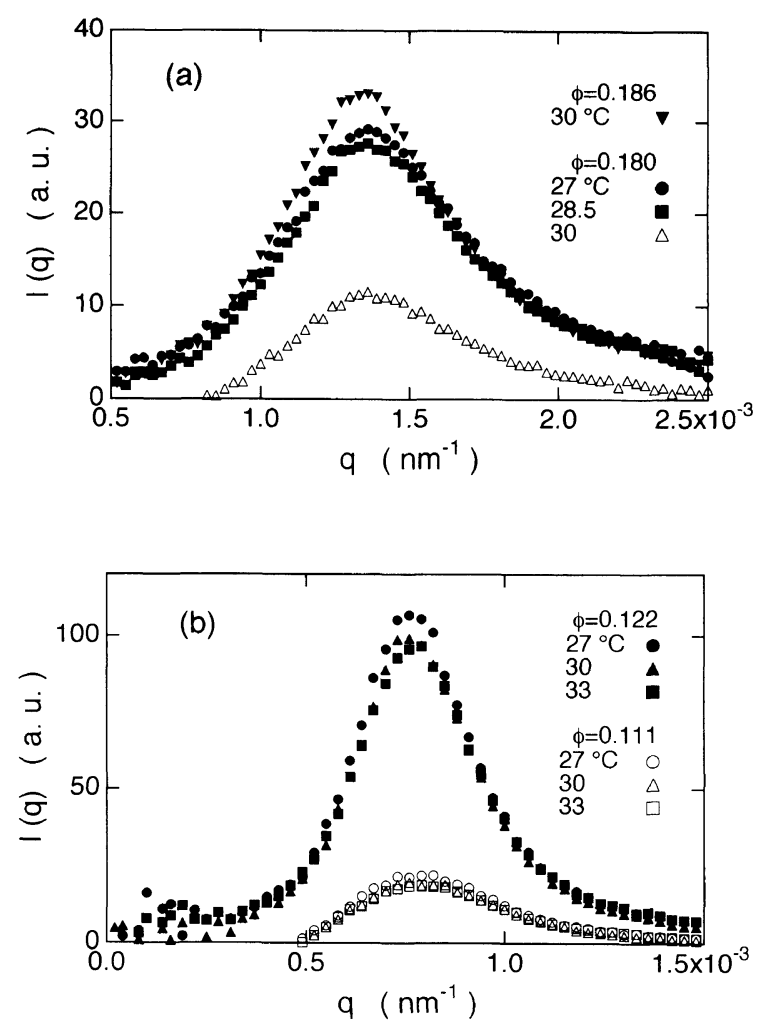

Figure 3. Plots of $I(q)$ vs. $q$ for (a) DP19 solutions and (b) DP20 solutions. Temperatures and volume fractions of DP19 are denoted in the figure. The filled and open symbols denote data in ordered and disordered states, respectively.

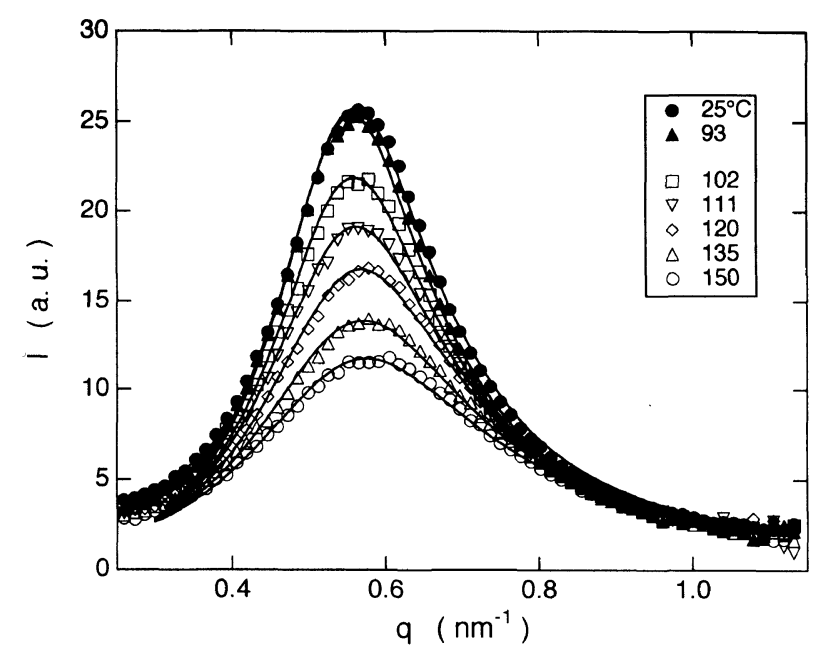

Figure 4. Plots of $I(q) v s . q$ for annealed sample of DP17 at different temperatures. Temperatures are denoted in the figure. Filled and open symbols denote data in ordered and disordered states, respectively. Solid lines denote fitting results to the Leibler's theory.

$\phi_{\mathrm{ODT}}$. As shown in Figure 5, $I^{*-1}$ and $D$ data can be expressed in terms of $T / T_{\mathrm{ODT}}$ using $T_{\mathrm{ODT}}$ thus obtained, for all samples mentioned above, though somewhat scattered.

A small decrease in $D$ in Figure $5 \mathrm{~b}$ and very shallow minimum of $I^{*-1}$ in Figure $5 \mathrm{a}$ are observed at $\left(T / T_{\mathrm{ODT}}\right)^{-1}$ slightly higher than 1 , which are most pronounced for $\phi=0.83$. This change in $D$ may be attributed to the glass transition of these samples. $D$ in the ordered state become higher due to volume expansion in the narrow range of $T, T_{\mathrm{g}}<T<T_{\mathrm{ODT}}$, than in glassy 

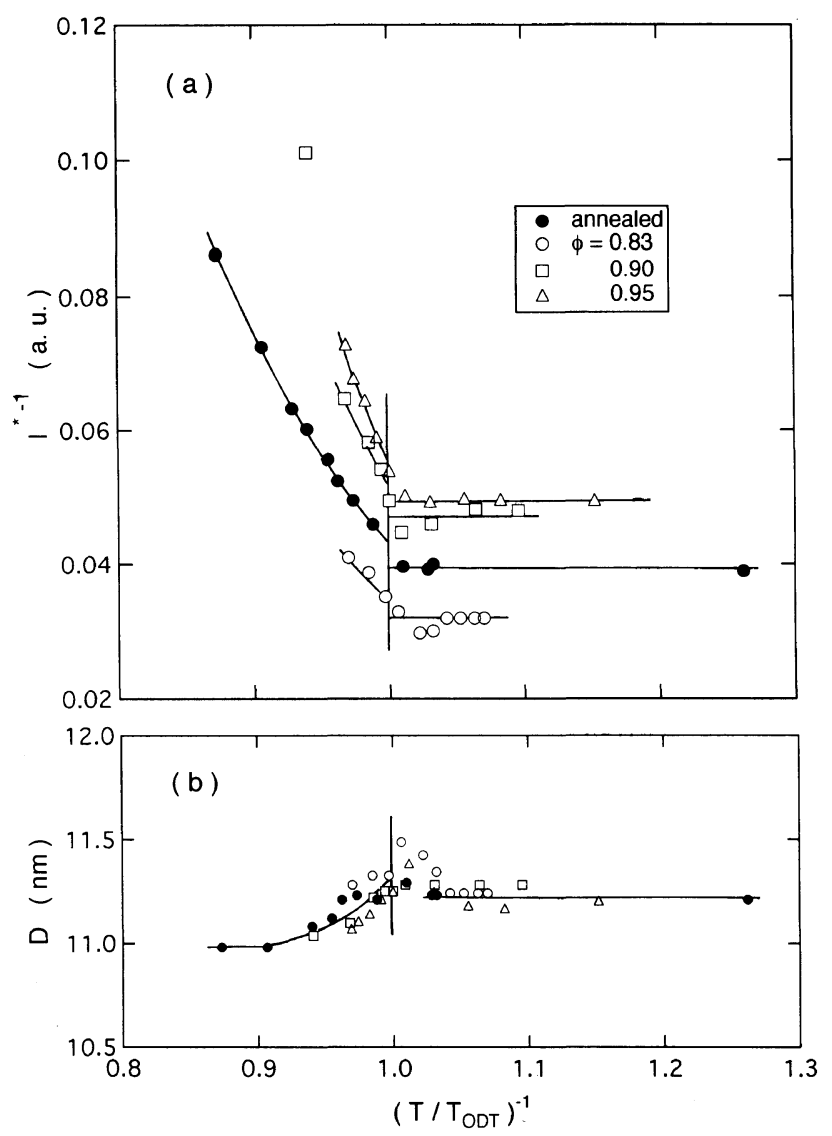

Figure 5. Plots of $I^{*-1}$ (a) and $D$ (b) against $\left(T / T_{\mathrm{ODT}}\right)^{-1}$ for DP17 in bulk and solutions. Symbols are denoted in the figure. The vertical solid lines denote $\left(T / T_{\mathrm{ODT}}\right)^{-1}=1$, while other lines are guides for eyes.

state since the $T_{\mathrm{g}} \mathrm{s}$ of these samples $\left(T_{\mathrm{g}}=57,53\right.$, and $39^{\circ} \mathrm{C}$ for $\phi=0.95,0.90$, and 0.85 , respectively) are slightly lower than the $T_{\mathrm{ODT}} \mathrm{s}$. The change in $I^{*-1}$ may be also correlated to glass transition.

\section{DISCUSSION}

In the disordered state, scattered profiles can be fitted to the theory of Leibler ${ }^{8}$ using $(\chi N)_{\text {eff }}$ and $\left\langle S^{2}\right\rangle^{1 / 2}$ as fitting parameters. The fitted values of $(\chi N)_{\text {eff }}$ may be different from the true values since the data are not desmeared, but the fitted values of $\left\langle S^{2}\right\rangle^{1 / 2}$ may be very close to the true value since the peak position of scattered profile is not seriously affected by the smearing effect. $\left\langle S^{2}\right\rangle^{1 / 2}$ evaluated for DP17 in bulk in the disordered states by the fitting procedure are almost constant around $3.3 \mathrm{~nm}$, close to the value of polystyrene with the same degree of polymerization in $\Theta$ solvents $(3.1 \mathrm{~nm}) .^{27}$

Almost the same fitting procedure can be used for DP19 and DP20 solutions in the disordered state using the floating base line and peak height as additional fitting parameters. If Leibler's theory is also applicable to these (semidilute) solutions, $\left\langle S^{2}\right\rangle^{1 / 2}$ can be evaluated. For example, the value evaluated for a DP20 solution $(\phi=0.111)$ by the fitting procedure is about $24.6 \mathrm{~nm}$, higher than the value in $\Theta$ solvents $(17.1 \mathrm{~nm})$, but close to $23.8 \mathrm{~nm}$ in good solvents at infinite dilution. ${ }^{27}$ Therefore, we conclude that the fitted results are close to the values at infinite dilution and further speculate that block copolymers in the disordered semidilute re-

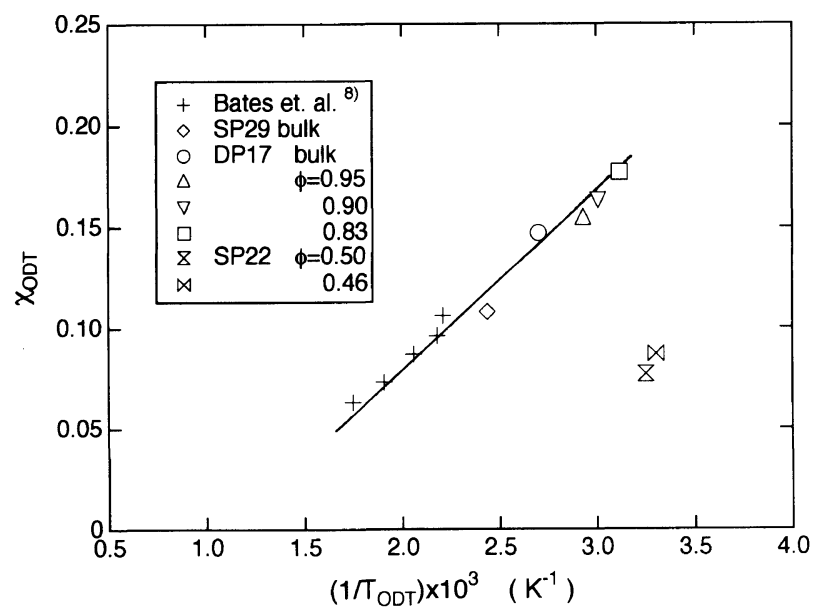

Figure 6. Plots of $\chi_{\text {ODT }} v s .1 / T_{\text {ODT }}$ for SP and DP diblock copolymers in bulk and solutions. Symbols are denoted in the figure. Solid line denotes inverse proportionality of $\chi$ on $T$ in melts and concentrated solutions.

gion are expanded in common good solvents from the unperturbed dimension by excluded volume effects, similarly to homopolymer solutions in the semidilute region, ${ }^{28}$ and also by fluctuation effect existing near ODT. ${ }^{3,5,7}$

Mayes et al. ${ }^{29}$ reported that the concentration dependence of the correlation length of the semidilute block copolymer solution is consistent with the scaling prediction in the semidilute region, whereas the concentration dependence of $q^{*}\left(\left\langle S^{2}\right\rangle^{1 / 2}\right)$ is weaker than the prediction. In this work, however, we do not discuss $\left\langle S^{2}\right\rangle^{1 / 2}$ further since the concentration range of the data is limited.

Finally we compare the observed ODT conditions with theory. It was reported ${ }^{3,23}$ that eq 1 holds for concentrated solutions if $(\chi N)_{\mathrm{ODT}}$ is replaced by $(\phi \chi N)_{\mathrm{ODT}}$. $\chi_{\text {ODT }}$ in bulk and solutions $(\phi>0.4)$ calculated from eq 1 are plotted against $T_{\text {ODT }}^{-1}$ in Figure 6 , together with the data reported by Bates et al. ${ }^{8}$ If $\chi$ is inversely proportional to $T$, the plot should give a straight line. Apparently, DP17 data in bulk and solutions fall on the straight line, but the data for SP22 solutions with $\phi=0.46$ and 0.50 do not, implying that the replacement of $(\chi N)_{\mathrm{ODT}}$ by $(\phi \chi N)_{\mathrm{ODT}}$ works well at highly concentrated solutions, but not around $\phi=0.5$.

For lower concentration solutions, the applicability of theory in the semidilute region was examined. Fredrickson and Leibler ${ }^{12}$ predicted that eq 1 also holds for the semidilute region if $\chi$ and $N$ (and $\bar{N}$ ) are replaced by $\phi_{\text {eff }} \sim \phi_{\chi \mathrm{SD}}$ and $\mathrm{Z} \sim N \phi^{1 /(3 v-1)}$ respectively, where $\chi_{\mathrm{SD}}$ is the crossover exponent and $v$ is the excluded volume exponent. Their theory predicts that the critical volume fraction for ODT varies with molecular weight as $\phi_{\text {ODT }} \sim$ $N^{-0.62}$ in common good solvents. A few experimental methods to test the theory were proposed in their paper. Since we cannot discuss the concentration dependence of the scattering profile, thus we compared the molecular weight dependence of $\phi_{\text {ODT }}$ with the theory at a constant temperature.

Figure 7 shows plots of $\phi_{\text {ODT }} v s$. $N$ with $\phi \leq 0.5$. As shown in Table II, the $\phi_{\mathrm{ODT}} \mathrm{s}$ of SP23, SP12, and DP20 were determined at $30^{\circ} \mathrm{C}$. Two values for DP19 were 


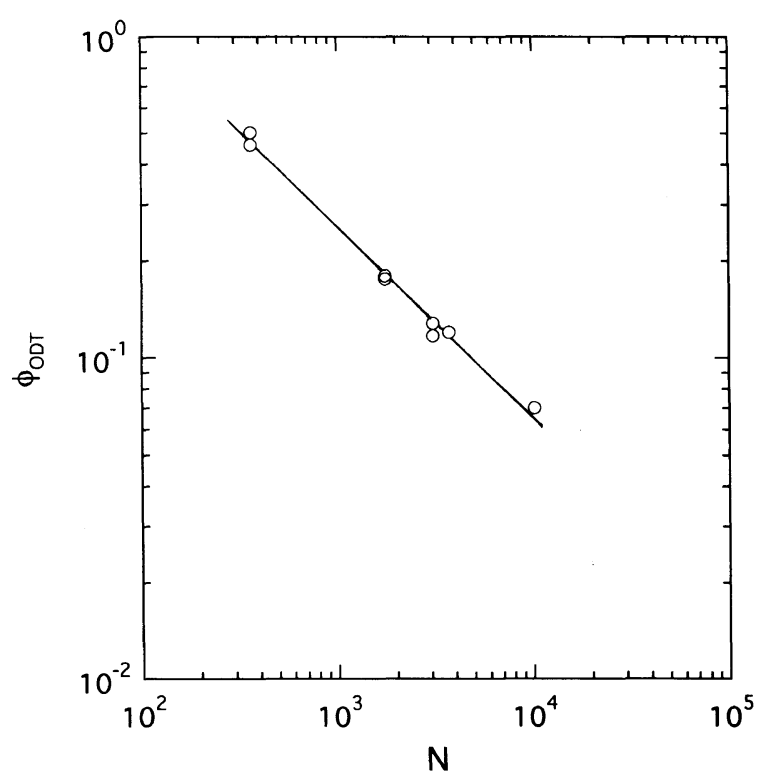

Figure 7. Double logarithmic plots of $\phi_{\mathrm{ODT}}$ against $N$ for SP and DP diblock copolymers in solutions. The solid line denotes the slope of -0.62 .

determined at temperatures very close to $30^{\circ} \mathrm{C}$, while two values for SP22 were determined at temperatures slightly lower and higher than $30^{\circ} \mathrm{C}$. Since temperature dependence is not significant as shown in Figure 7, we can safely assume that these data with $\phi \leq 0.5$ are at constant $T\left(30^{\circ} \mathrm{C}\right)$. The solid line denotes the slope of -0.62 , agrees with the data up to $\phi=0.5$. Therefore, the relationship between $\phi_{\mathrm{ODT}}$ and $N$ may be explained by the theory in the semidilute region. However, more systematic studies are needed for definite conclusion, since the concentration range where the semidilute behavior holds is extended to much higher concentration than that of ordinary polymer solutions where the scaling law is applicable to thermodynamic properties, and the crossover concentration from semidilute to concentrated regimes is not clear in this work.

Acknowledgments. We thank Dr. Alan Nakatani et al. at National Institute of Standards and Technology in Gaithersburg MD, U.S.A., for assistance in the preliminary SANS measurements and valuable discussion. This work was supported by a Grant-in-Aid for Scientific Research (No. 09650994) from the Ministry of Education, Science, and Culture of Japan.

\section{REFERENCES AND NOTES}

1. T. Hashimoto, in "Thermoplastic Elastomers," N. R. Legge, G. Holden, and H. E. Schroeder, Ed., Hanser, Munich, 1987, Chapter 3.

2. F. S. Bates and G. H. Fredrickson, Annu. Rev. Phys. Chem., 41, 525 (1990).

3. T. P. Lodge, X. Xu, X. Jin, and M. C. Dalvi, Macromol. Symp., 79, 87 (1994).

4. F. S. Bates, M. F. Schulz, A. K. Khandpur, S. Förster, J. H. Rosedale, K. Almdal, and K. Mortensen, Faraday, Discuss., 98, 7 (1994).

5. J. H. Rosedale, F. S. Bates, K. Almdal, K. Mortensen and G. D. Wignall, Macromolecules, 28, 1429 (1995).

6. N. Sakamoto and T. Hashimoto, Macromolecules, 28, 6825 (1995).

7. T. Ogawa, N. Sakamoto, T. Hashimoto, C. D. Han, and D. M. Beak, Macromolecules, 29, 2113 (1996).

8. M. F. Schulz, A. K. Khandpur, F. S. Bates, K. Almdal, K. Mortensen, D. A. Hajduk, and S. M. Gruner, Macromolecules, 29, 2857 (1996).

9. L. Leibler, Macromolecules, 13, 1602 (1980).

10. G. H. Fredrickson and E. J. Helfand, Chem. Phys., 87, 697 (1987).

11. M. Olivera de la Cruz, J. Chem. Phys., 90, 1995 (1989).

12. G. H. Fredrickson and L. Leibler, Macromolecules, 22, 1238 (1989).

13. Y. Takahashi, M. Noda, N. Ochiai, and I. Noda, Polymer, 37, 5943 (1996).

14. Y. Matsushita, K. Shimizu, Y. Nakao, H. Choshi, I, Noda, and M. Nagasawa, Polym. J., 18, 361 (1986).

15. Y. Takahashi, N. Ochiai, Y. Matsushita, and I. Noda, Polym. J., 28, 1065 (1996).

16. Y. Matsushita, Y. Nakao, R. Saguchi, H. Choshi, and M Nagasawa, Polym. J., 18, 493 (1986).

17. Y. Matsushita, Y. Nakao, K. Shimizu, I. Noda, and M Nagasawa, Macromolecules, 21, 2790 (1988).

18. Y. Matsushita, Y. Nakao, R. Saguchi, K. Mori, H. Choshi, Y. Muroga, I. Noda, M. Nagasawa, T. Chang, C. J. Glinka, and C. C. Han, Macromolecules, 21, 1802 (1988).

19. Y. Matsushita, K. Mori, R. Saguchi, Y. Nakao, I. Noda, and M. Nagasawa, Macromolecules, 23, 4313 (1990).

20. Y. Matsushita, K. Mori, Y. Mogi, R. Saguchi, I. Noda, M Nagasawa, T. Chang, C. J. Glinka, and C. C. Han, Macromolecules, 23, 4317 (1990).

21. Y. Mogi, H. Kotsuji, Y. Kaneko, K. Mori, Y. Matsushita, I. Noda, Polym. J., 23, 227 (1991).

22. Y. Matsushita, K. Shimizu, I. Noda, T. Chang, and C. C. Han, Polymer, 33, 2412 (1992).

23. T. Hashimoto, M. Shibayama, and H. Kawai, Macromolecules, 16, 1093 (1983).

24. N. P. Balsara, B. Hammouda, P. K. Kesani, S. V. Jonnalagadda, and G. C. Straty, Macromolecules, 27, 2566 (1994).

25. This SANS measurements was performed with the $8 \mathrm{~m}$ SANS spectrometer at National Institute of Standards and Technology in Gaithersburg, MD, U.S.A.

26. F. S. Bates, J. H. Rosedale, and G. H. Fredrickson, J. Chem. Phys., 92, 6255 (1990).

27. Y. Matsushita, I. Noda, M. Nagasawa, T. P. Lodge, E. J. Amis, and C. C. Han, Macromolecules, 17, 1785 (1984).

28. M. Daoud, J. P. Cotton, B. Farnoux, G. Jannink, G. Sarma, H. Benoit, R. Duplessix, C. Picot, and P. G. De Gennes, Macromolecules, 8, 804 (1975).

29. A. M. Mayes, J. G. Barker, and T. P. Russel, J. Chem. Phys., 101, 5213 (1994). 